\title{
Bifosfonatos en Odontopediatría: Revisión de la literatura, protocolo de manejo y reporte de un caso clínico
}

\author{
Ingrid Báez Madrigal, ${ }^{1}$ \\ Rubí López Fernández, ${ }^{2}$ \\ Jorge Pedro Téllez Rodríguez, ${ }^{2}$
}

\section{Resumen}

En la actualidad, los bifosfonatos son utilizados en la población pediátrica con diagnóstico de osteogénesis imperfecta, desórdenes neuromusculares, displasia ósea, displasia fibrosa, osteoporosis idiopática juvenil, enfermedad de Crohn y trastornos reumatológicos. La osteonecrosis de los maxilares secundaria al tratamiento con bifosfonatos, se ha convertido en una complicación cada vez más común debido al incremento de su uso en la población adulta. En cuanto a la atención pediátrica la información es escasa. Este estudio presenta el caso clínico de una paciente de sexo femenino con diagnóstico de osteogénesis imperfecta tipo III quien fue tratada con múltiples extracciones dentales bajo anestesia general, previa administración de amoxicilina $50 \mathrm{mg} / \mathrm{kg}$ 1 hora antes; debido a erupción anómala de caninos y primeros premolares superiores, así como persistencia de caninos y molares primarios superiores. Se realizaron citas control postoperatorias, en donde mostró adecuado proceso de cicatrización sin datos de necrosis maxilar. El objetivo es informar al estomatólogo sobre cómo actúan los bifosfonatos, los diagnósticos en la población pediátrica tratados con dicho fármaco y dar a conocer el protocolo de manejo de estos pacientes. Se realizó una revisión integradora en las bases de datos PubMed y Cochrane Library, los artículos fueron seleccionados de acuerdo a los criterios establecidos.

Palabras clave: Cirugía oral, pediatría, bifosfonato. 
Caso clinico

\section{Bisfosfonatos em Odontopediatria: Revisão da literatura, protocolo de gestão e relato de caso clínico}

\section{Resumo}

Atualmente, os bisfosfonatos são utilizados na população pediátrica diagnosticada com osteogênese imperfeita, distúrbios neuromusculares, displasia óssea, displasia fibrosa, osteoporose idiopática juvenil, doença de Crohn e distúrbios reumatológicos. A osteonecrose dos maxilares secundárias ao tratamento com bifosfonatos tornou-se uma complicação cada vez mais comum devido ao aumento do uso na população adulta. Quanto ao atendimento pediátrico, as informações são escassas. Este estudo apresenta o caso clínico de uma paciente do sexo feminino com diagnóstico de osteogênese imperfeita tipo III, tratada com múltiplas exodontias sob anestesia geral, após administração de amoxicilina $50 \mathrm{mg} / \mathrm{kg}$
1 hora antes; devido à erupção anômala de caninos superiores e primeiros prémolares, bem como persistência de caninos decíduos superiores e molares. Foram marcadas consultas de controle pós-operatório, onde apresentava processo de cicatrização adequado, sem dados de necrose maxilar. O objetivo é informar ao estomatologista como os bisfosfonatos atuam, os diagnósticos na população pediátrica tratada com esse medicamento e apresentar o protocolo de manejo desses pacientes. Realizou-se revisão integrativa nas bases de dados PubMed e Cochrane Library, os artigos foram selecionados de acordo com os critérios estabelecidos.

Palabra-chave: Cirurgia maxilofacial, pediatria, bifosfonato.

Case report

\section{Bisphosphonates in pediatric dentistry: literature review, management protocol and report of a clinical case}

\begin{abstract}
Bisphosphonates are currently used in the pediatric population diagnosed with osteogenesis imperfecta, neuromuscular disorders, skeletal dysplasia, fibrous dysplasia, juvenile idiopathic osteoporosis, Crohn's disease, and rheumatologic disorders. Bisphosphonate-related osteonecrosis of the jaw has become an increasingly common complication due to its increased use in the adult population. Regarding pediatric care, information is scarce. This study presents the clinical case of a female patient
\end{abstract}

diagnosed with type III osteogenesis imperfecta who was treated with multiple dental extractions under general anesthesia, after administration of amoxicillin $50 \mathrm{mg} / \mathrm{kg}$ 1 hour before; due to anomalous eruption of upper canines and first premolars, as well as persistence of upper primary canines and molars. Postoperative control appointments were made, where she showed an adequate healing process without evidence of maxillary necrosis. The aim is to inform the stomatologist about how bisphosphonates act, the diagnoses in the pediatric population treated with 
this drug and to present the management protocol for these patients. An integrative review was carried out in the PubMed and Cochrane Library databases, the articles

\section{Introducción}

El hueso es una forma especial de tejido conectivo fisiológicamente mineralizado, que se renueva constantemente en respuesta a hormonas, fuerzas mecánicas y envejecimiento; está formado por células vivas y muertas incrustadas en la matriz orgánica mineralizada, compuesta de colágeno tipo I, proteoglicanos y proteínas no colagenosas. ${ }^{1,2}$

La remodelación ósea permite que el tejido óseo responda a la reparación mecánica de carga y evite microdaños, mantiene el control de la calidad ósea y permite la liberación de factores de crecimiento y minerales almacenados durante toda la vida. $^{2}$

En la infancia la remodelación ósea es crítica para la erupción dental y durante la dentición permanente, la erupción requiere de reabsorción de las raíces de la dentición decidua y del hueso coronal al órgano dentario en desarrollo. Debido a la acción de los bifosfonatos en la inhibición de la resorción ósea, el potencial de estos agentes para retrasar la erupción dental es de interés. ${ }^{3}$ En los pacientes pediátricos con diagnóstico de osteogénesis imperfecta (OI), existe una alteración en la síntesis de la matriz ósea y la homeostasis induciendo la fragilidad ósea. El tratamiento farmacológico es mediante el uso de bifosfonatos, los cuales tienen efectos sobre las propiedades mecánicas del hueso debido a que modifican la remodelación ósea. ${ }^{4}$ were selected according to the established criteria.

Key words: Oral surgery, pediatrics, bisphosphonate.

\section{Método}

Se llevó a cabo una revisión de la literatura especializada mediante las bases de datos PubMed y Cochrane Library utilizando la terminología MeSH. Los criterios de inclusión establecidos para la selección de los artículos son: artículos que tras la búsqueda bibliográfica y lectura se adapten al tema a tratar, artículos que posean según su metodología calidad alta de evidencia, artículos que debido a su relevancia para el estudio y calidad científica deben ser incluidos a pesar de no cumplir con los criterios de inclusión anteriores. Los criterios de exclusión establecidos son: imposibilidad de obtener el artículo a texto completo, no adaptarse al tema a tratar, artículos que de acuerdo a su metodología posean una calidad media o baja de evidencia.

\section{Bifosfonatos}

Los bifosfonatos, son una clase de compuestos químicos activos farmacológicos que inhiben la acción de los osteoclastos y la resorción ósea. Son análogos estables del pirofosfato inorgánico, se depositan debajo de los osteoclastos y en el hueso recién formado. ${ }^{2}$

Todos los bifosfonatos se caracterizan por 2 grupos de fosfonato que comparten de esqueleto un átomo de carbono común (P-C-P), responsable de la fuerte afinidad de los bifosfonatos por el mineral óseo, 
la hidroxiapatita. Los bifosfonatos simples unidos al calcio son absorbidos por los osteoclastos por endocitosis y se incorporan como metabolitos tóxicos no hidrolizables, análogos de ATP o que contienen metileno. Los metabolitos análogos de ATP contienen los grupos $\mathrm{P}-\mathrm{C}-\mathrm{P}$ de bifosfonatos en lugar del resto pirofosfato (P-O-P) de ATP.

Los análogos de ATP son resistentes a la descomposición hidrolítica y a la liberación de fosfato. Los metabolitos de los bifosfonatos simples, pueden incorporarse al sitio activo de la enzima aminoacil-ARNt sintetasa en la célula.

Estos metabolitos citotóxicos se condensan y se acumulan en el citosol de los osteoclastos, causando su apoptosis.

En resumen, los bifosfonatos simples actúan como profármacos, absorbidos por los osteoclastos donde se acumulan como metabolitos tóxicos, causan apoptosis de los osteoclastos y evitan la resorción ósea. ${ }^{5}$

Los bifosfonatos que contienen nitrógeno (N-bifosfonatos), como el Risedronato, Ibandronato, Pamidronato, Alendronato, Ácido Zolendrónico y Minodronato ${ }^{5}$ son más potentes que los bifosfonatos simples e inhiben los osteoclastos de diferente manera; interrumpen la formación de osteoclastos, la supervivencia y la dinámica del citoesqueleto, mientras que los no nitrogenados inician la apoptosis osteoclástica. ${ }^{5,7}$

\section{Uso de bifosfonatos en el paciente pediátrico}

El tratamiento con bifosfonatos en pacientes pediátricos fue sugerido en
1998, cuando la administración cíclica del pamidronato intravenoso en niños con OI, resultó en la disminución de la incidencia de fracturas. ${ }^{5}$

Además de su uso en la OI, también se ha descrito en la Histiocitosis de células de Langerhans, displasia fibrosa, desórdenes neuromusculares, displasia ósea, osteoporosis idiopática juvenil, osteomielitis multifocal crónica recurrente (OMCR), trastornos reumatológicos y en la enfermedad de Crohn. ${ }^{3,5,8}$

El régimen de tratamiento con bifosfonatos y la duración del tratamiento son variables, dependen del diagnóstico y la respuesta al tratamiento. El pamidronato tiende a administrarse a una dosis de $9 \mathrm{mg} /$ $\mathrm{kg} / \mathrm{año}$, administrados en intervalos de entre 1 y 4 mensuales. La dosis de ácido zoledrónico tiende a ser de $0,1 \mathrm{mg} / \mathrm{kg} / \mathrm{año}$, a intervalos de entre 3 y 12 mensuales. En OI y osteoporosis, la mayoría de los niños serán tratados durante al menos dos años y muchos necesitan terapia continua. La duración del tratamiento para la displasia fibrosa y la OMCR varía con la respuesta al dolor, pero la mayoría de los niños reciben tratamiento durante al menos 12 meses. $^{3}$

Los efectos adversos reportados con mayor frecuencia asociados al tratamiento de bifosfonatos en niños son síntomas similares a la gripe, irritación gastrointestinal, esofagitis química, dolor óseo y/o muscular, uveítis transitoria, hipocalcemia. $^{3}$

En adultos, los bifosfonatos parecen suprimir la resorción ósea hasta 10 años después de la interrupción, en los niños más pequeños debido a mayor recambio óseo, la recuperación de los bifosfonatos 
reciclados del hueso es más corta. Los niños con OI tratados con bifosfonatos a temprana edad tienen un crecimiento normal o mejorado y se informa sobre la adquisición de hueso nuevo en estudios de fracturas de huesos largos después de la interrupción del tratamiento con pamidronato intravenoso. Así mismo, se reporta el aumento de tamaño de los huesos vertebrales y reconfiguración de fracturas de compresión vertebral preexistentes. $^{5}$

\section{Protocolo recomendado para manejo estomatológico del paciente pediátrico en tratamiento con bifosfonatos}

La falta de pautas para el tratamiento quirúrgico oral que aborden específicamente a los pacientes pediátricos tratados con estos agentes, ha llevado a implementar protocolos destinados para adultos, como regímenes de antibióticos profilácticos en niños. De acuerdo a los datos de la experiencia en adultos, la característica clave para adquirir necrosis de los maxilares (NM) es traumatismo de los maxilares (extracción dental o cirugía dento alveolar) y un historial de exposición prolongado a altas dosis de N-bifosfonatos. El uso cada vez mayor de estos agentes en niños y la variación en regímenes profilácticos, requieren de pautas específicas para el tratamiento quirúrgico oral de los niños bajo tratamiento con bifosfonatos intravenosos. Las recomendaciones fueron desarrolladas de acuerdo a una evaluación de la literatura existente, así como de acuerdo a la opinión de los expertos del Working Party del Children's Hospital en Westmead en Australia. $^{3}$
Principios generales para la prevención de NM en niños:

1. Evaluación odontológica integral y eliminación de focos infección oral antes del tratamiento con bifosfonatos.

2. Educación del paciente para el mantenimiento de salud bucal óptima.

3. Realizar procedimientos dentales invasivos previo al tratamiento con bifosfonatos. Tres semanas deben ser consideradas para permitir tiempo adecuado de cicatrización.

4. Aplazamiento de la terapia con bifosfonatos hasta que se optimice la salud oral, o al menos hasta que se hayan curado los sitios quirúrgicos.

\section{Recomendaciones para procedimientos quirúrgicos orales en niños bajo tratamiento con bifosfonatos:}

a) En niños donde las infusiones de bifosfonatos ya han comenzado, los órganos dentarios de mal pronóstico deben extraerse lo más tarde posible en el ciclo de infusión, permitiendo un mínimo de 3 semanas antes de la próxima infusión, en coordinación con el médico tratante del paciente.

b) Realizar enjuagues con gluconato de clorhexidina al $0,12 \% 2$ veces al día durante 5 días antes del procedimiento, minutos antes del procedimiento y durante 5 días 2 veces al día después del procedimiento.

c) Se recomienda pausar el tratamiento con bifosfonatos (Drug Holiday) de 3 
a 6 meses, en aquellos pacientes que serán sometidos a cirugía oral invasiva. Decisión tomada por médico tratante..$^{3,9-11}$

d) No existe contraindicación para el uso de anestésico local con vasoconstrictor, ya que el efecto del mismo es transitorio.

e) En el caso de extracciones o cirugía bucal es necesario eliminar bordes filosos o espículas óseas.

f) Se sugiere utilización de sutura reabsorbible con agujas atraumáticas o con el corte externo con técnica de colchonero horizontal o en $\mathrm{X}$.

g) Realizar revisiones periódicas durante los 12 meses postoperatorios.

h) Se recomienda profilaxis antibiótica en aquellos pacientes de alto riesgo de acuerdo a las características descritas a continuación. ${ }^{12}$ (Tabla 1 )

Tabla 1: Régimen de Profilaxis Antibiótica. ${ }^{3}$

\begin{tabular}{|l|l|l|l|}
\hline Situación & Agente & Dosis & Tiempo \\
\hline $\mathrm{IV}^{*}$ & Ampicilina & $\begin{array}{l}50 \\
\mathrm{mg} / \mathrm{kg}\end{array}$ & $\begin{array}{l}\text { Previo a } \\
\text { procedimiento }\end{array}$ \\
\hline VO $^{* *}$ & Amoxicilina & $\begin{array}{l}50 \\
\mathrm{mg} / \mathrm{kg}\end{array}$ & $\begin{array}{l}1 \text { hr. previa a } \\
\text { procedimiento }\end{array}$ \\
\hline $\begin{array}{l}\text { Alergia a } \\
\text { Penicilina } \\
\text { IV }\end{array}$ & Clindamicina & $\begin{array}{l}15 \\
\mathrm{mg} / \mathrm{kg}\end{array}$ & $\begin{array}{l}\text { Previo a } \\
\text { procedimiento }\end{array}$ \\
\hline $\begin{array}{l}\text { Alergia a } \\
\text { Penicilina } \\
\text { VO }\end{array}$ & Clindamicina & $10 \mathrm{mg}$ & $\begin{array}{l}1 \text { hr. previa a } \\
\text { procedimiento }\end{array}$ \\
\hline
\end{tabular}

*IV: Intravenoso, **VO: Vía Oral

\section{Factores de riesgo en el paciente pediátrico con bifosfonatos:}

1. Tratamiento quirúrgico invasivo (extracciones múltiples, curetajes, fijación de fracturas) o procedimientos traumáticos.

2. Absceso o fístula de tejido blando asociado, evidencia radiográfica de patología periapical que indique contaminación bacteriana significativa del hueso alveolar.

3. Haber recibido infusiones por 2 o más años y que la última infusión sea en los últimos 2 años para pacientes menores de 14 años o en los últimos 5 años para pacientes mayores de 14 años.

4. Inflamación periodontal y/o tratamiento concomitante con quimioterapia o corticosteroides. ${ }^{3}$

\section{Caso clínico}

Paciente femenino de 11 años de edad con diagnóstico de OI tipo III, con antecedente de 31 fracturas durante su toda su vida, bajo tratamiento con Vitamina D3 1600 unidades y $1 \mathrm{mg}$ de ácido zoledrónico por más de 2 años, última aplicación hace 9 meses. En el momento de su tratamiento la paciente se encontraba cursando quinto año de primaria con un buen desarrollo en el colegio. A la exploración se encuentra activa, reactiva, potencialmente cooperadora. Facies triangular, biotipo facial euriprosopo, asimetría facial, cejas arqueadas, órbita izquierda más superior 

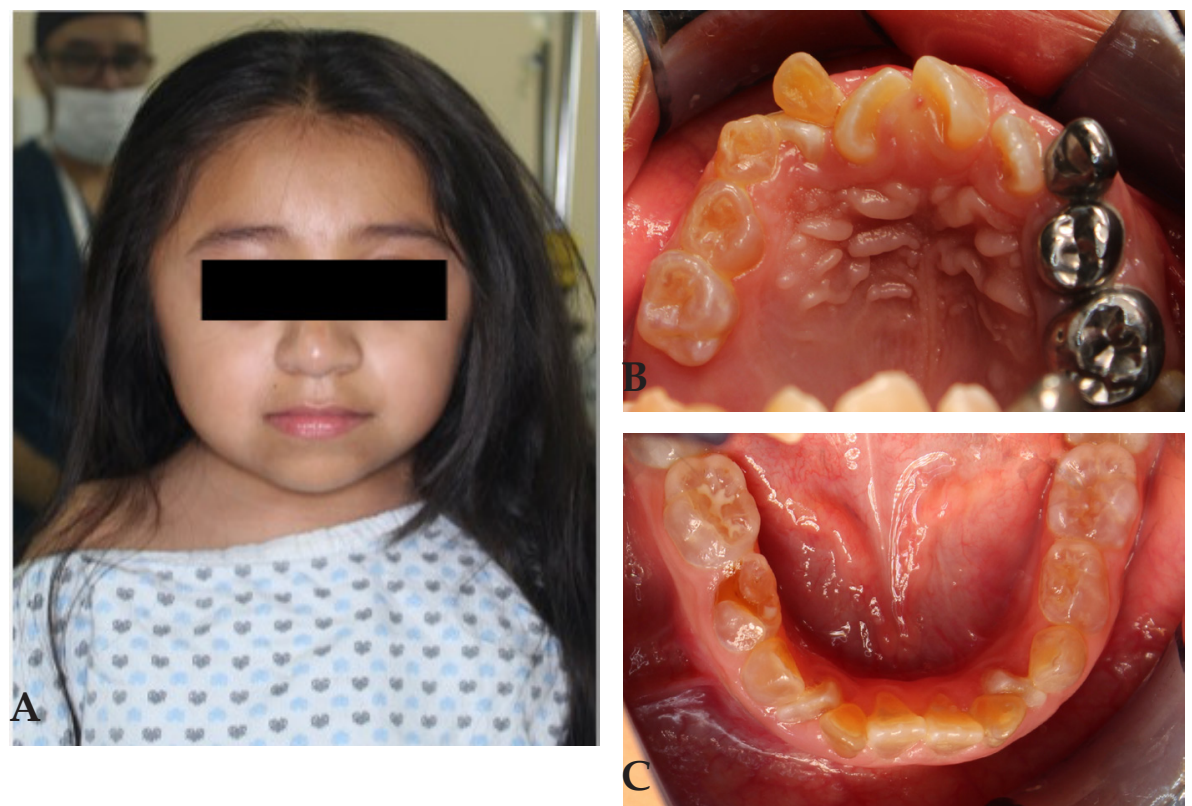

Figura 1: Fotografías iniciales. A. Fotografía facial. B. Fotografía oclusal superior. C. Fotografía oclusal inferior

que órbita derecha, puente nasal ancho y deprimido, fisuras palpebrales rectas, escleras azules, mentón prominente. Relación 1:2 subnasal-estomion-menton, labio superior e inferior delgados. Intraoralmente mucosas íntegras de adecuada coloración, dentición mixta de color opalescente libre de caries, con retraso en la erupción y maloclusión clase III de Angle. Perteneciente al grupo de alto riesgo para caries de acuerdo a CAMBRA. (Figura 1: A,B,C)
Radiográficamente, vía de erupción anómala de caninos y primeros premolares superiores, así como persistencia de caninos y molares primarios superiores, trazo sugerente de fractura antigua en región parasinfisiaria izquierda. (Figura 2:A, B) El departamento de Estomatología del Instituto Nacional de Pediatría de acuerdo a un análisis clínico y radiográfico, diagnosticó edad dental de 8,3 de acuerdo al método de Demirjian, falta de espacio para erupción de la dentición permanente,
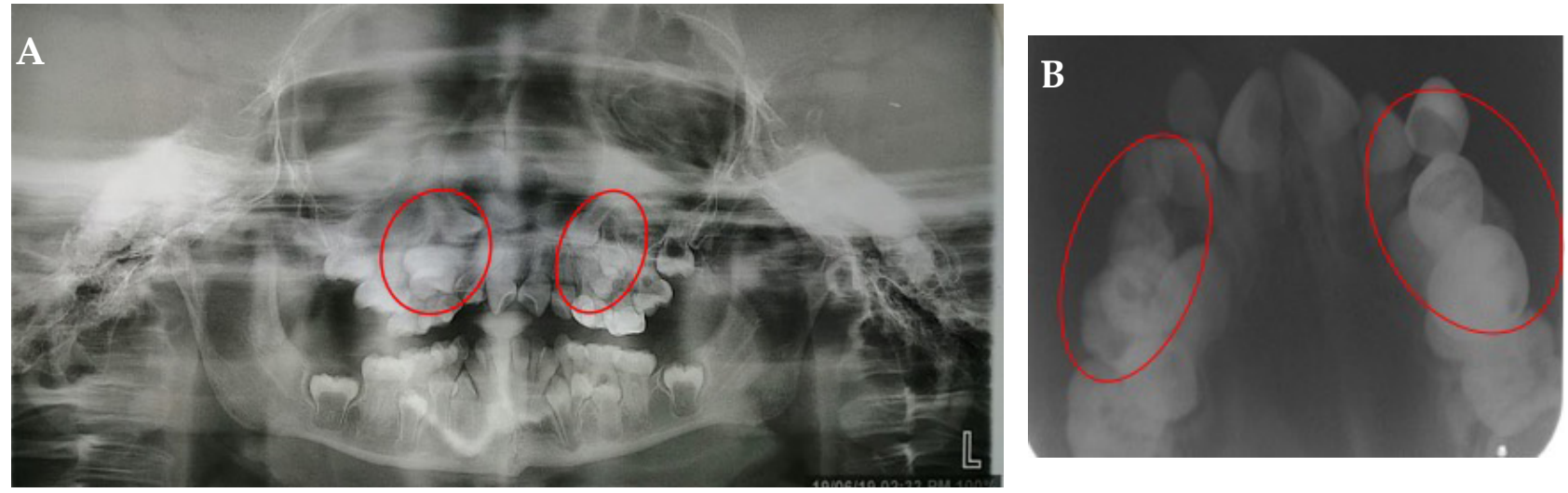

Figura 2: Radiografías: A. Panorámica. B. Oclusal 

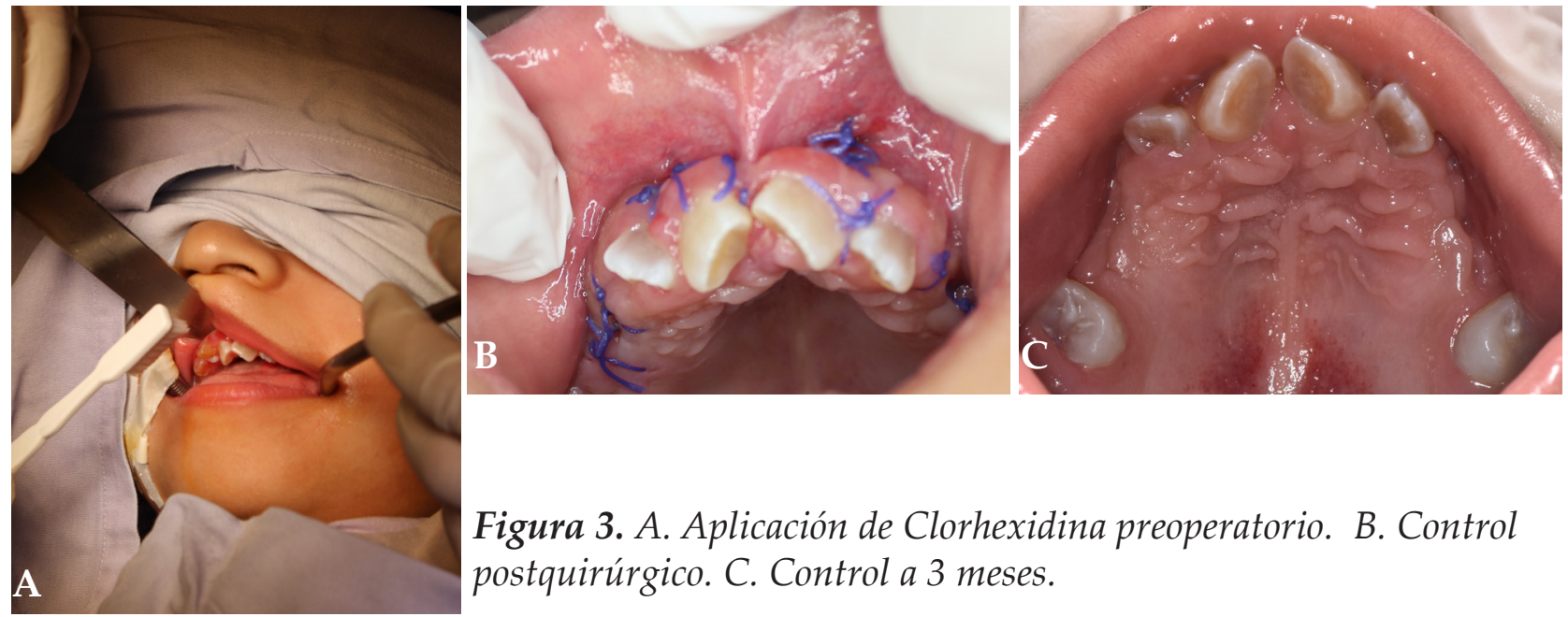

Figura 3. A. Aplicación de Clorhexidina preoperatorio. B. Control postquirúrgico. C. Control a 3 meses. vía de erupción anómala de caninos y primeros premolares, riesgo de resorción externa por presión a los órganos dentarios erupcionados; presencia de diversas persistencias dentales superiores, se decidió tratamiento con extracciones de dichos órganos dentarios. En la arcada inferior no fue llevado a cabo ningún tratamiento, debido a no presentar vía de erupción anómala. Se pautó plan de tratamiento con Cirugía Maxilofacial, se interconsultó al servicio de Endocrinología, quienes indicaron pausa en el tratamiento (drug holiday) de 3 meses. Previa firma de consentimiento informado por parte de sus padres, se administró amoxicilina $50 \mathrm{mg} / \mathrm{kg} 1$ hora antes, bajo anestesia general, con intubación naso traqueal, se realizó doble colgajo semi Newman para osteotomía, extracción de los caninos y molares primarios superiores y enucleación de los caninos y primeros premolares superiores. Previo a la cirugía, se le indicó a la paciente realizar enjuagues con gluconato de clorhexidina al $0,12 \% 2$ veces al día durante 5 días y de igual manera postoperatoriamente. (Figura 3:A)

Se realizaron citas control postoperatorias durante 3 meses, mostrando adecuado proceso de cicatrización sin datos de NM o infección. (Figura 3: B, C)

\section{Discusión}

En la actualidad, no existen reportes de casos de NM en niños o adolescentes. ${ }^{13-16}$ Brown et al. examinaron 42 pacientes pediátricos que se encontraban en tratamiento con bifosfonatos, quienes fueron examinados clínicamente $\mathrm{y}$ radiográficamente para descartar NM, a 11 de estos pacientes se les realizaron tratamientos dentales invasivos y no fueron encontrados signos o síntomas relacionados con NM. ${ }^{14}$

En un estudio por Chanine et al, 278 pacientes recibieron al menos un ciclo de pamidronato intravenoso, 113 fueron tratados con extracciones dentales durante el tratamiento con pamidronato. Se determinó que ninguno de los pacientes tenía antecedentes sugestivos de NM. ${ }^{15}$

Un grupo de 15 pacientes con OI a quienes se les realizaron extracciones dentales en el Hospital de Niños de Montreal entre 2000 y 2006 fue revisado por Schwartz et al. Se realizaron 60 extracciones, el $65 \%$ 
durante tratamiento activo con bifosfonatos intravenosos. El 23\% después de completar el tratamiento con bifosfonatos. Ninguno de los casos desarrolló infección, retardo en la cicatrización ni NM. ${ }^{16}$

En un estudio con ratas se ha encontrado retraso en la erupción dental, secundario a la administración de bifosfonatos, coincidiendo con nuestro caso clínico. En dos estudios se encontraron, defectos del esmalte y áreas de anquilosis entre el hueso alveolar y los tejidos duros dentales en desarrollo. ${ }^{17-19}$ Estos hallazgos se han atribuido a la detención de la remodelación ósea y alteración de actividades osteoclásticas inducida por bifosfonatos. ${ }^{3}$

En cuanto a la ortodoncia el impacto de los bifosfonatos es de interés, se ha reportado mayor tiempo en el tratamiento, aumentando las probabilidades de un cierre deficiente del espacio y del paralelismo radicular. ${ }^{20}$ De acuerdo a Ghoneima et al., una preocupación adicional es el aumento del recambio óseo alveolar como resultado del tratamiento de ortodoncia, pudiendo aumentar la captación de bifosfonatos localmente en la mandíbula, incrementando el riesgo de desarrollo de NM en el futuro. ${ }^{21}$

El esqueleto pediátrico no se puede comparar directamente con el esqueleto adulto, ya que el recambio y la dinámica ósea son muy diferentes, esto hace que las comparaciones de dosis de bifosfonatos intravenosos y NM sean complejas. La vida media depende de la tasa de recambio óseo, ya que los huesos trabeculares tienen una tasa de recambio más alta que el hueso cortical y los bifosfonatos tienen una mayor afinidad por los huesos con tasas de recambio altas. Esto explica el por que la mandíbula es el sitio mas frecuente de NM debido a su alta tasa de recambio óseo, siendo esta de 3 a 5 veces mas activa a nivel alveolar que a nivel del canal mandibular en adultos. ${ }^{22}$

Debido a que el uso de bifosfonatos en niños es reciente en comparación con los adultos, hay muy poca información sobre los potenciales efectos adversos a largo plazo. La literatura existente es bastante consistente en medidas rigurosamente preventivas para todos los niños tratados con estos agentes, particularmente por el desconocimiento de las potenciales complicaciones a largo plazo. ${ }^{9}$

\section{Conclusión}

El estomatólogo pediatra y cirujanos maxilofaciales que se encuentren en contacto con pacientes bajo tratamiento con bifosfonatos deben estar pendientes de los tratamientos médicos, manteniéndose en comunicación con el médico tratante, conocer los potenciales riesgos y el manejo correcto. Es recomendable advertir a los padres el potencial riesgo de NM, así como incluirlo en el consentimiento informado. Así mismo es importante incorporar a los padres y al paciente a un programa preventivo de acuerdo al nivel de riesgo de lesiones de caries en el que se encuentre y realizar un estricto control de erupción para evitar alteraciones.

Se ha demostrado el beneficio de los bifosfonatos en niños, por lo que su uso ha ido en incremento. Los estudios que investigan la existencia de NM en niños tratados con bifosfonatos son retrospectivos, con pequeñas muestras y pocos; sin embargo, estos estudios apoyan demostrando que no existe antecedente de NM en niños. 
Nuestras recomendaciones pretenden dar guía de acuerdo al juicio del estomatólogo para cada caso individual. Debido a la escasa existencia de literatura se requieren más estudios a futuro para mejorar el conocimiento sobre los riesgos potenciales y permitir complementar el protocolo o modificarlo de ser necesario.

\section{Referencias bibliográficas}

1. Gasser JA, Kneissel M. Bone Physiology and Biology. En: Smith SY, Varela A, Samadfam R. Bone Toxicology. XII ed. Montreal, Canada. Springer International 2017:27-94.

2. Dominguez L, Di Bella G, Belvedere M, Barbagallo M. Physiology of the aging bone and mechanisms of action of bisphosphonates. Biogerontology 2011;12:397-408. DOI 10.1007/s10522-011-9344-5

3. Batt RN, Hibbert SA, Munns CF. The use of bisphosphonates in children: review of the literature and guidelines for dental management. Aust Dent J 2014;59:9-19. DOI: 10.1111/adj.12140

4. Bourgeois AB, Aubry-Rozier B, Bonafé L, et al. Osteogenesis imperfecta: from diagnosis and multidisciplinary treatment to future perspectives. Swiss Med Wkly 2016;146:1

5. Eghbali-Fatourechi G. Bisphosphonate therapy in pediatric patients. J Diabetes Metab Disord. 2014;13:109.

6. Endo Y, Kumamoto H, Nakamura M, et al. Underlying Mechanisms and Therpeutic Strategies for Bisphosphonate-Related Osteonecrosis of the jaw (BRONJ). Biol. Pharm. Bull 2017;40:739-750.

7. Dwan K, Phillipi CA, Steiner RD, Basel D. Bisphosphonate therapy for osteogenesis imperfecta. Cochrane Database of Systematic Reviews 2016;10:1-71. DOI: 10.1002/14651858.CD005088.pub4.

8. Ngan K-K, Bowe J, Goodger N. The Risk of Bisphosphoate Related Osteonecrosis of the Jaw in Children. A Case Report and Literature Review. Dent Update 2013;40:733-738.

9. Khosla S, Burr D, Cauley J, et al. Bisphosphonate-associated osteonecrosis of the jaw: report of a task force of the American Society for Bone and Mineral Research. J Bone Miner Res 2007;22:1479-1491.

10. Khan AA, Sandor GK, Dore E, et al. Canadian consensus practice guidelines for bisphosphonate associated osteonecrosis of the jaw. J Rheumatol 2008;35:1391-1397.

11. Ruggiero SL, Dodson TB, Assael LA, Landesberg R, Marx RE, Mehrotra B. American Association of Oral and Maxillofacial Surgeons position paper on bisphosphonate-related osteonecrosis of the jaws 2009 update. J Oral Maxillofac Surg 2009;67:2-12.

12. Sambrook P, Olver I, Goss A. Bisphosphonates and osteonecrosis of the jaw. Aust Fam Physician 2006;35:801-803.

13. Malmgren B, Astrom E, Soderhall S. No osteonecrosis in jaws of young patients with osteogenesis imperfecta treated with bisphosphonates. J Oral Pathol Med 2008; 37:196-200.

14. Brown JJ, Ramalingam L, Zacharin MR. Bisphosphonate associated osteonecrosis of the jaw: does it occur in children? Clin Endocrinol (Oxf) 2008;68:863-867.

15. Chahine C, Cheung MS, Head TW, Schwartz S, Glorieux FH, Rauch F. Tooth extraction socket healing in pediatric patients treated with intravenous pamidronate. J Pediatr 2008;153:719-720.

16. Schwartz S, Joseph C, Iera D, Vu D-D. Bisphosphonates, osteonecrosis, osteogenesis imperfecta and dental extractions: a case series. J Can Dent Assoc 2008;74:537-542.

17. Grier RL, Wise GE. Inhibition of tooth eruption in the rat by a bisphosphonate. J Dent Res 1998;77:8-15.

18. Bradaschia-Correa V, Massa LF, Arana-Chavez VE. Effects of alendronate on tooth eruption and molar root formation in young growing rats. Cell Tissue Res 2007;330:475-485.

19. Hiraga T, Ninomiya T, Hosoya A, Nakamura H. Administration of the bisphosphonate zoledronic acid during tooth development inhibits tooth eruption and formation and induces dental abnormalities in rats. Calcif Tissue Int 2010;86:502-510.

20. Lotwala RB, Greenlee GM, Ott SM, Hall SH, Huang GJ. Bisphosphonates as a risk factor for adverse orthodontic outcomes: a retrospective cohort study. Am J Orthod Dentofacial Orthop 2012;142:625-634.

21. Ghoneima AA, Allam ES, ZuntSL, Windsor LJ. Bisphosphonates treatment and orthodontic considerations. Orthod Cranio- fac Res 2010;13:1-10.

22. Hennedige AA, Jayasinghe J, Khajeh J, Macfarlane TV. Systematic Review on the Incidence of Bisphosphonate Related Osteonecrosis of the Jaw in Children Diagnosed with Osteogenesis Imperfecta. J Oral Maxillofac Res 2013;4(4):e1 Disponible en: http://www.ejomr.org/JOMR/archives/2013/4/e1/ v4n4e1ht.pdf

Recibido: 30/06/2020

Aceptado: 18/11/2020

Correspondencia: Ingrid Báez Madrigal, correo: ingridinpediatria@gmail.com 